\title{
A NOTE ON SYSTEMS OF LINEAR EQUATIONS ${ }^{1}$
}

\section{David L. Elliott ${ }^{2}$}

This NOTE Is A COMMENT on reference [1] and a generalization of the method there presented. We consider a system of $m$ linear equations in $n$ unknowns $x_{1}, x_{2}, \cdots, x_{n}$,

$$
\sum_{j=1}^{n} a_{i j} x_{j}=c_{i} \quad i=1,2, \cdots, m, a_{i j}, c_{i} \text { real }
$$

or $A \cdot x=c$ in matrix notation. We distinguish three cases:

(I) There is no finite vector $x$ satisfying (1) (inconsistent case);

(II) There is a unique vector $x$ satisfying (1);

(III) There are an infinity of vectors $x$ satisfying (1), such that their endpoints lie on some line, plane, or higher-dimensional linear manifold.

Semarne considers Case II for $m=n$, but the presentation in reference [1] requires modification, as will be shown below.

Semarne's method of solving (1) combines the two classical ideas of the augmented-matrix equation and Gram-Schmidt orthogonalization. (The method does not involve computing a matrix inverse to $A$.) As modified in this report, the method will handle any system (1) whether or not $A$ has an inverse, furnishing a solution if one exists and informing us if one does not exist.

We shall use vectors (or points)

$$
x=\left(x_{1}, x_{2}, \cdots, x_{n}\right)
$$

in $E^{n}$ (euclidean $n$-space),

$$
y=\left(y_{0}, y_{1}, \cdots, y_{n}\right)
$$

in $E^{n+1}$. We extend $E^{n}$ by introducing, in addition to the $x_{i}$, an artificial variable $t$. Multiplying (1) by $t$ and transposing, we obtain:

$$
-c_{i} t+\sum_{j=1}^{n} a_{i j} t x_{j}=0 \quad i=1,2, \cdots, m,
$$

or

$$
\sum_{j=0}^{n} b_{i j} y_{j}=0 \quad i=1,2, \cdots, m
$$

where

$$
b_{i 0}=-c_{i}, \quad b_{i j}=a_{i j} \quad j=1,2, \cdots, n,
$$

and

$$
y_{j}=t x_{j}, 1 \leqq j \leqq n \text {, and } y_{0}=t .
$$

1 Received by the editors August 10, 1959.

2 U. S. Naval Ordnance Test Station, Pasadena Annex. 
(This notation differs from that of reference [1].) If $x$ is a solution of (1), then for arbitrary $t(3)$ gives a solution of (2) lying on a line through the origin of $E^{n+1} ;\left(1, x_{1}, \cdots, x_{n}\right)$ is the intersection of this line with the hyperplane $y_{0}=1$. Conversely, if we find any point $y^{*}$ satisfying (2), then every point on the line $y=s y^{*},-\infty<s<\infty$, also satisfies (2). If $y_{0}^{*} \neq 0$, let $s^{*}=1 / y_{0}^{*}$; for the point $y=s^{*} y^{*}, y_{0}=1$. Then in (3) we have $t=y_{0}=1$,

$$
s^{*} y_{j}^{*}=y_{j}=x_{j}, \quad 1 \leqq j \leqq n,
$$

and $x$ satisfies (1).

If $y_{0}{ }^{*}=0$, the line $y=s y^{*}$ lies in the hyperplane $y_{0}=0$ and can have no finite intersection with $y_{0}=1$, so no corresponding solution of (1) can be found. If $y_{0}=0$ for every $y$ satisfying (2), we classify (1) as Case I, inconsistent. We shall give explicit forms for solutions of (2) and (1) below.

Equation (2),

$$
B \cdot y=0,
$$

where $B$ is the $m$ by $n+1$ matrix $\left(b_{i j}\right)$, can be interpreted geometrically in two ways. The first is that the vector $y$ lies in the intersection of $m$ hyperplanes (each passing through the origin). That is, the "solution" of (2) is a vector subspace, $U$, of $E^{n+1}$ : the origin itself, or some line, plane, etc., through the origin. Defining the vector $\left(b_{i 1}, b_{i 2}, \cdots, b_{i n}\right)=b^{i}$ for any $i=1,2, \cdots, m$, each hyperplane has equation

$$
b^{i} \cdot y=0 \quad i=1,2, \cdots, m .
$$

Then the vector $b^{i}$ is perpendicular to the $i$ th hyperplane. Our second interpretation of $(2)$ is "any solution-vector $y$ is perpendicular to the $m$ vectors $b^{1}, \ldots, b^{m}$," and a "solution" is the subspace $U$ which can be described as the orthogonal complement in $E^{n+1}$ of the subspace $V$ spanned by $b^{1}, \cdots, b^{m}$. (That is, any point in $V$ can be written as a linear combination of the $b^{i}$.) Then we need to find a set of vectors which span $U$, in order to define the solution of (2) completely.

From the viewpoint of the second interpretation, above, a good solution method is evident, namely, the Gram-Schmidt orthogonalization procedure. We note these facts:

a. One complete basis of orthogonal vectors for $E^{n+1}$ is the set

$$
\{u\}=\left\{\begin{array}{l}
u^{0}=(1,0, \cdots, 0,0), \\
u^{1}=(0,1, \cdots, 0,0), \\
\ldots \ldots \ldots \cdots \cdots \\
u^{n}=(0,0, \cdots, 0,1) .
\end{array}\right.
$$

b. We can form an orthogonal basis for $V$, consisting of $M$ mutually orthogonal vectors $(M \leqq m) f^{1}, f^{2}, \cdots f,{ }^{M}$, from the (not necessarily independent) vectors $b^{1}, \cdots, b^{m}$ by using the Gram-Schmidt process. 
c. If we continue the Gram-Schmidt process by adjoining vectors-say, from $\{u\}$-which do not belong to $V$, we eventually will obtain a new complete orthogonal basis for $E^{n+1}$,

$$
f^{1}, f^{2}, \cdots, f^{M}, f^{M+1}, \cdots, f^{n+1},
$$

and the vectors (if any) $f^{M+1}, \cdots, f^{n+1}$ will be a complete orthogonal basis for $U$, hence a "solution". If $M=n+1, U$ contains only the origin.

As Semarne points out, the beauty of this approach is that the Gram-Schmidt computation is quite simple; the basis vectors are:

$$
\begin{aligned}
f^{1} & =b^{1} \\
f^{2} & =b^{2}-\left(\frac{f^{1} \cdot b^{2}}{f^{1} \cdot f^{1}}\right) f^{1} \\
\ldots \ldots \ldots \ldots \ldots \ldots & \ldots \ldots \\
f^{m} & =b^{m}-\left(\frac{f^{1} \cdot b^{m}}{f^{1} \cdot f^{1}}\right) f^{1}-\left(\frac{f^{2} \cdot b^{m}}{f^{2} \cdot f^{2}}\right) f^{2}-\cdots-\left(\frac{f^{m-1} \cdot b^{m}}{f^{m-1} \cdot f^{m-1}}\right) f^{m-1} .
\end{aligned}
$$

Now $M(\leqq m)$ of these vectors are non-zero; we may omit the others and renumber the non-zero ones as $f^{1}, \cdots, f^{M}$; they span $V$. Now we continue computing until we obtain a total of $n+1$ non-zero vectors:

$$
\begin{aligned}
f^{M+1}= & u^{0}-\left(\frac{f^{1} \cdot u^{0}}{f^{1} \cdot f^{1}}\right) f^{1}-\cdots-\left(\frac{f^{M} \cdot u^{0}}{f^{M} \cdot f^{M}}\right) f^{M} \\
= & u^{0}-\left(\frac{f_{0}^{1}}{f^{1} \cdot f^{1}}\right) f^{1}-\cdots-\left(\frac{f_{0}{ }^{M}}{f^{M} \cdot f^{M}}\right) f^{M}, \\
f^{M+2}= & u^{1}-\left(\frac{f_{1}{ }^{1}}{f^{1} \cdot f^{1}}\right) f^{1}-\cdots-\left(\frac{f_{1}{ }^{M+1}}{f^{M+1} \cdot f^{M+1}}\right) f^{M+1} \\
& \quad \ldots \ldots \cdots-\left(\frac{f_{n}{ }^{n}}{f^{n} \cdot f^{n}}\right) f^{n},
\end{aligned}
$$

where again we have dropped all $f$ 's which are zero. The "solution" of (2) is then the space $U$ consisting of all $y$ such that:

$$
y=\alpha_{M+1} f^{M+1}+\cdots+\alpha_{n+1} f^{n+1} \text {, for any real } \alpha_{i} .
$$

We can obtain the solution of (1) as the intersection (if any) of $U$ with the hyperplane $y_{0}=1$; that is, points of $U$ having $y_{0}=1$ satisfy (1). We can now, from the vectors of $\left(5^{\prime}\right)$, decide which case (of the first paragraph) we have:

(I) If $f_{0}{ }^{K}=0$ for all the $f^{K}$ in $\left(5^{\prime}\right), U$ lies in the hyperplane $y_{0}=0$, and no finite solution of (1) exists.

(II) If it happens that $M=n$ and that $f_{0}{ }^{n+1} \neq 0$, (6) becomes $y=\alpha_{n+1} f^{n+1}$; we take $s_{0}=1 / f_{0}{ }^{n+1}$ and write:

$$
s_{0} y=\left(1, x_{1}, \cdots, x_{n}\right) ;
$$

$x$ solves (1) uniquely. (See note below.) 
(III) If $M<n$ and some $f_{0}{ }^{K} \neq 0, K>M$, (2) is satisfied, from (6), by

$$
\bar{y}=\frac{\sum_{K=M+1}^{n+1} \alpha_{K} f^{K}}{\sum_{K=M+1}^{n+1} \alpha_{K} f_{0}{ }^{K}} \text { whenever } \sum_{K=M+1}^{n+1} \alpha_{K} f_{0}{ }^{K} \neq 0 .
$$

It is evident that $\bar{y}_{0}=1$, so $\bar{y}=\left(1, x_{1}, \cdots, x_{n}\right)$ and $x$ solves $(1)$ if the conditions of (7) are satisfied. Thus, (1) is completely solved.

NotE. Reference [1] considers only Case II, for $m=n$, but the author forms $f^{n+1}$ not from $u^{0}$, but from his vector:

$$
e^{n+1}=(1,1, \cdots, 1,1) .
$$

This vector is unsatisfactory for the following reason. In reference [1], the matrix $A$ of (1) is assumed $n$-by- $n$ non-singular; then no combination of its row vectors $a^{i}$ can satisfy:

$$
\sum_{i=1}^{n} \beta_{i} a^{i}=0, \quad \sum_{i=1}^{n} \beta_{i} \neq 0 .
$$

Then no combination of the $b^{i}$ of the matrix $B$ can equal $u^{0}$, so $f^{n+1} \neq 0$ and we obtain our solution. But if we replace $u^{0}$ with $e^{n+1}$ in the orthogonalization it may occur that, for a non-singular $A$, some combination of the rows of $B$ will equal $e^{n+1}$; then we get a zero solution of (2), and the solution of (1) is indeterminate, as in the example:

$$
\begin{aligned}
2 x_{1}+x_{2} & =-2, \\
x_{1}+0 & =-1 .
\end{aligned}
$$

In such a case, reference [1] gives $f^{3}=(0,0,0)$, whereas our $f^{3}=\left(\frac{1}{2},-\frac{1}{2}, 0\right)$, giving the solution $x_{1}=-1, x_{2}=0$.

\section{REFERENCE}

1. H. Manvel Semarne, New direct method of solution of a system of simultaneous linear equations, SIAM Review, 1, 1(1959), pp. 53-54. 